P. Brekke, B. Fleck, and J. B. Gurman eds.

\title{
Soft X-ray Flares and Magnetic Configuration in Solar Active regions
}

\section{H. Zhang}

Beijing Astronomical Observatory National Astronomical Observatories Chinese Academy of Sciences, Beijing 100012, China

\begin{abstract}
In this paper, we analyze the soft X-ray flares and the relationship with photospheric vector magnetograms and current helicity in solar active regions.
\end{abstract}

\section{Non-potential magnetic field and current helicity}

The strong magnetic shear in the active regions normally connects with solar flares. It is reflects the twisted magnetic lines of force or the interaction of the different system of the magnetic fields.

Helicities are topologically a measure of the structural complexity of the corresponding fields. The helicity of magnetic fields may be characterized by several different parameters. The magnetic helicity density $h_{m}=\mathbf{A} \cdot \mathbf{B}$, with $\mathbf{A}$ the vector potential for magnetic field $\mathbf{B}$ (i.e. $\mathbf{B}=\nabla \times \mathbf{A}$ ), measures the chirality of magnetic lines of force. The total magnetic helicity in a volume $V$ is

$$
H_{m}=\int_{V} \mathbf{A} \cdot \mathbf{B} d^{3} x
$$

which may not be conserved when finite resistivity is present. However, such magnetic helicity is unmeasurable in the solar atmosphere immediately.

We notice that the current helicity is another quality for studying the twist of magnetic field, which can be written in the form

$$
H_{c}=\int_{V} \mathbf{B} \cdot(\nabla \times \mathbf{B}) d^{3} x .
$$

The current helicity density can be written into two parts,

$$
h_{c}=\mathbf{B}_{\|} \cdot(\nabla \times \mathbf{B})_{\|}+\mathbf{B}_{\perp} \cdot(\nabla \times \mathbf{B})_{\perp} .
$$

The first term in the right side of equation (3) is observationable, and can be inferred by photospheric vector magnetograms. It is important to provide the basic property of the twisted magnetic field near strong magnetic poles in the active regions, because one normally believes that the magnetic poles vertically extend up from the deep atmosphere.

In the approximation of the force free field,

$$
h_{c}=\alpha B^{2}=\left(B / B_{\|}\right)^{2} \mathbf{B}_{\|} \cdot(\nabla \times \mathbf{B})_{\|},
$$



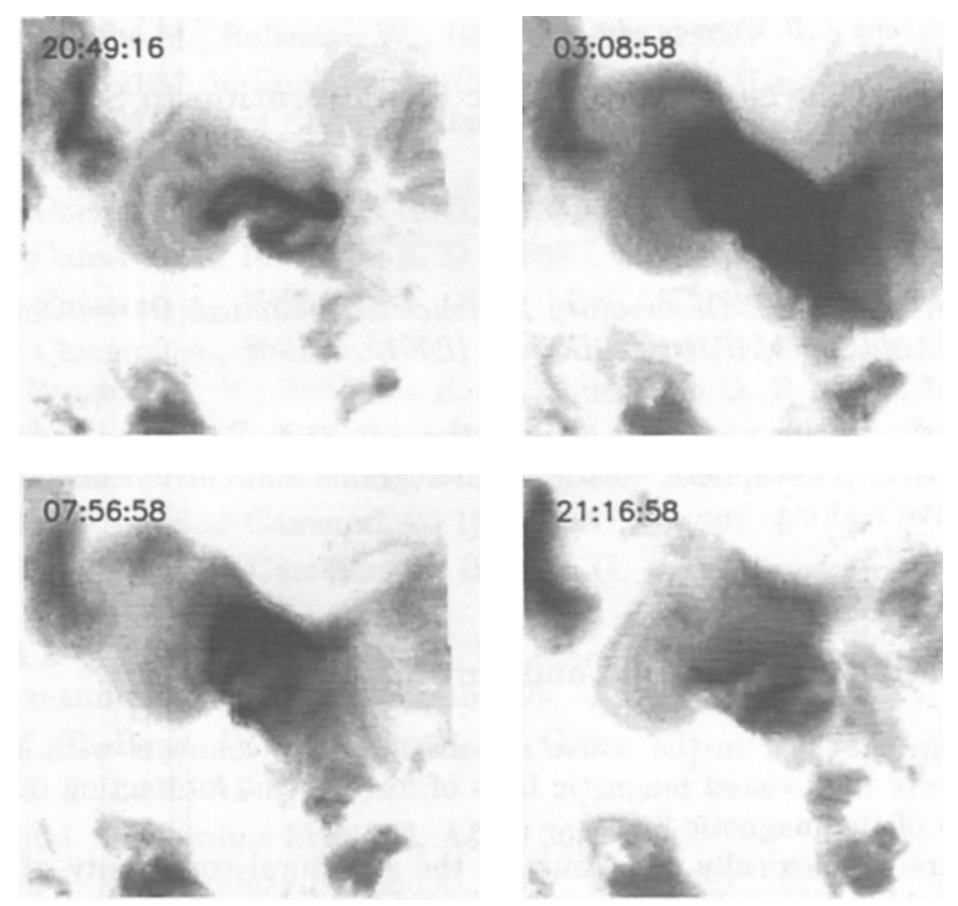

Figure 1. A time sequence of soft X-ray images of the M4.9/2B flare on February 24-25, 1992. The size of images is 13 'times 13'.

where $\alpha$ is not constant. If $\alpha$ is constant, the simple relationship between the current and magnetic helicity densities can be found,

$$
h_{c}=\alpha^{2} h_{m} .
$$

As we study the variation of the current helicity by using Faraday's low $\frac{\partial \mathbf{B}}{\partial t}=-c \nabla \times \mathbf{E}$, the evolution of the current helicity density can be derived,

$$
\frac{\partial h_{c}}{\partial t}=-2 c \nabla \times \mathbf{E} \cdot \nabla \times \mathbf{B}-c \nabla \cdot((\nabla \times \mathbf{E}) \times \mathbf{B}) .
$$

It is found that the current helicity is not conserved.

\section{Current helicity and flares}

Figure 1 shows a series of soft X-ray images of the M4.9/2B flare on February 24-25, 1992 in the active region (NOAA 7070) near the center of the solar disk. We can see that the soft X-ray loops quickly spread all over the space at the high solar atmosphere in the active region after the pre-flare stage, which provides the distribution of the soft X-ray flare loops in the top of view (Zhang et al. 2000). 


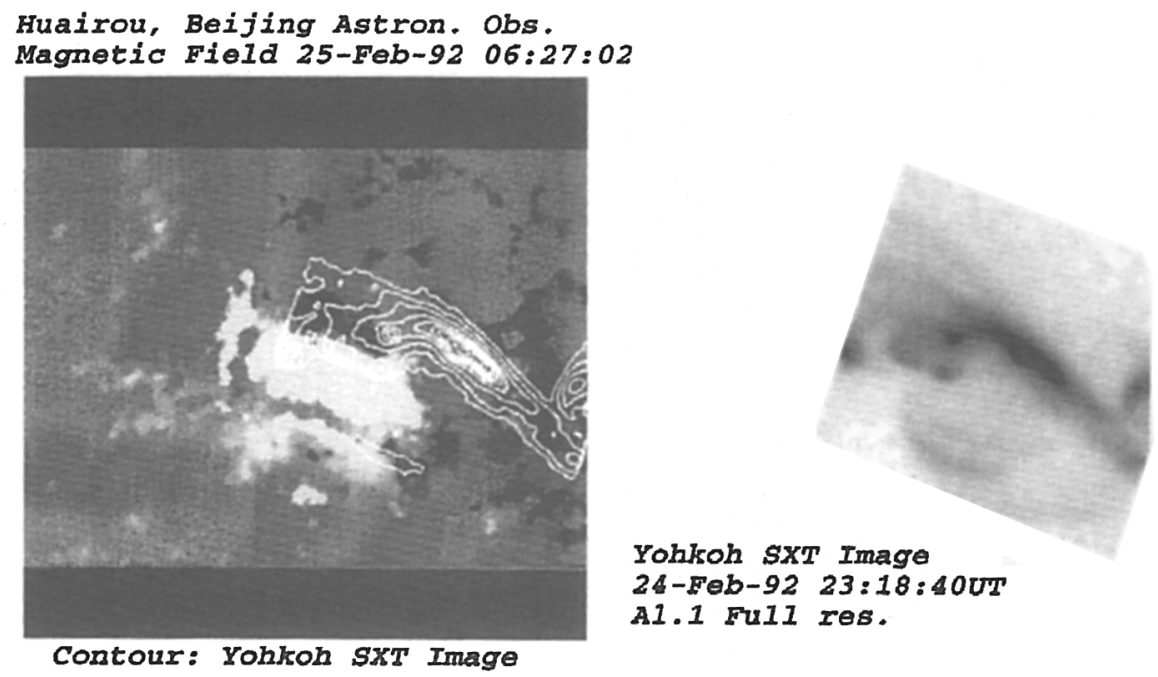

Figure 2. A longitudinal magnetogram (left), overlaid by the soft Xray image (right) by contour lines on February 25, 1992. The size of magnetogram is $4 . ' 5$ times $3 . ' 4$.

As one overlaps a soft X-ray image on the longitudinal magnetogram on February 25, 1992 in Figure 2, we can find that the bright loop-like structure during the pre-flare phase in soft X-ray images connected the magnetic pole $N_{1}$ of positive polarity with the enhanced network magnetic field of negative polarity. Although the overlay between the soft X-ray image and magnetogram is adjusted by the photospheric image in the Yohkoh satellite, the soft X-ray image actually reflects the information of the hooter plasma, or rather, does not reflect the footpoints rooted in the cooler photosphere. This provides a basic relationship between the soft X-ray features and the photospheric magnetic field during the pre-flare phase in the active region.

Figure 3 shows a vector magnetogram on February 25, 1992. The $180^{\circ}$ ambiguity of the transverse component of the magnetic field is normally resolved by the approach of the potential field. The corresponding current helicity density parameter $\mathbf{B}_{\|} \cdot(\nabla \times \mathbf{B})_{\|}$in Figure 3 shows that the basic topology of twist magnetic field. We can easily find that the local current helicity in the photosphere of the active region can be obviously separated into two parts, if we follow the continuity of the photospheric transverse field. One shows negative sign and connects with the sheared magnetic field region $\left(N_{1}\right.$ and $\left.S_{1}\right)$, and another shows positive sign and is located near the magnetic pole $N_{2}$. If we follow the direction of the photospheric transverse magnetic field and the sign of the electric current, the region with the positive helicity also contains the magnetic pole $S_{2}$ and probably the surrounding enhanced magnetic network near the magnetic pole $S_{2}$. This means that two parts with opposite signs of the electric current helicity in the active region show oppositely twisted characteristics of the magnetic 

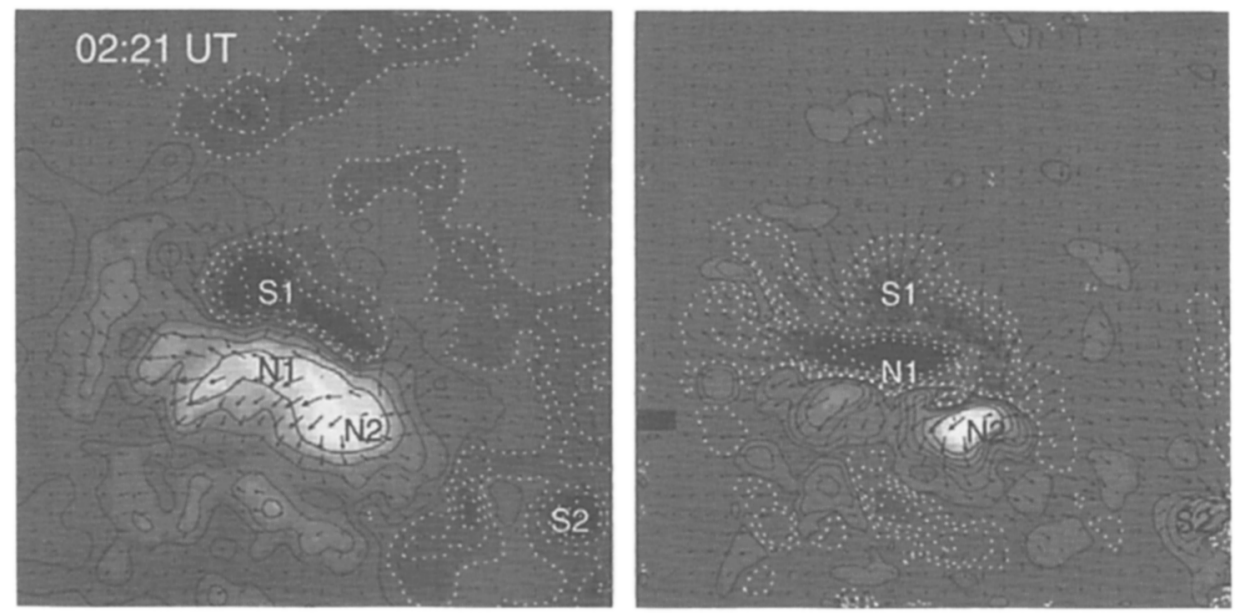

Figure 3. A photospheric vector magnetogram (left) and the corresponding current helicity parameter $\mathbf{B}_{\|} \cdot(\nabla \times \mathbf{B})_{\|}$overlapping by the transverse magnetic field (right) in the active region 7070 on February 25,1992 . The arrows mark transverse magnetic field. The white and black areas with contours mark the positive and negative polarity respectively.

field. The flares in the active region (NOAA 7070) were triggered by different magnetic loops systems, i.e. the pre-flares occurred near the interface of the magnetic fields with opposite (or different) directions.

\section{References}

Zhang H., Sakurai T., Shibata K., Shimojo M. \& Kurokawa H. 2000, A\&A 357, 725 